SHORT REPORT

\title{
Advice given to patients with epistaxis by A\&E doctors
}

\author{
N Eze, S Lo, A Toma
}

Emerg Med J 2005;22:724-725. doi: 10.1136/emj.2004.015842

This study was devised to determine the proportion of patients with epistaxis seen in accident and emergency (A\&E) departments and discharged with verbal/written advice, and to examine if this information affects re-attendance rates. A questionnaire was given to all A\&E doctors probing their current practice regarding advice given to patients with epistaxis on discharge. The information was complemented with a case note audit of patients with epistaxis seen and discharged by A\&E doctors over a 2 month period, which assessed the content and format of advice given and the number of re-attendances. The standard used stated that all patients discharged from A\&E should be given both verbal and written advice regarding the prevention and management of further bleeds. The A\&E doctors were then invited to a presentation on the management of epistaxis and the appropriate advice to give patients on discharge. Written advice leaflets on the prevention and management of further bleeds were placed in the A\&E department and were accessible to doctors and patients. Case notes were reaudited over the following 2 month period. Verbal and written advice increased from $19 \%$ to $61 \%$ and $2 \%$ to $54 \%$ respectively. The number of re-attenders who had previously only seen A\&E doctors was reduced from 11 (17\%) in the first half to $5(8 \%)$ in the second half of the cycle, representing a $9 \%$ reduction. We conclude that the provision of adequate verbal and written advice to patients with epistaxis may have an important role in the prevention of further bleeds and subsequent re-attendance to $A \& E$ departments.

M any patients with epistaxis are seen in the A\&E department by casualty doctors. A substantial proportion is discharged without seeing an ear, nose, and throat (ENT) doctor because their bleeds are not deemed severe enough for admission. Many are given limited information on management of further bleeds and even fewer are advised on the prevention of bleeds.

The aims of this audit were to examine the information given to patients with epistaxis who are seen and discharged by $A \& E$ doctors, to instigate an improvement in current practice, and to examine the subsequent effect on reattendance rates. The standard used suggests that, on discharge, all patients with epistaxis should be given both written and verbal advice regarding avoidance of further bleeds and appropriate action to take should a bleed recur. ${ }^{12}$

\section{METHODS}

A questionnaire was distributed to all A\&E doctors probing their current practice regarding epistaxis advice given to patients. The data obtained were complemented by examination of true practice by assessment of the A\&E notes of patients presenting in March/April 2003. The number of reattenders was also noted.

The A\&E doctors were then invited to a presentation given by the ENT department on the management and prevention of epistaxis and the appropriate verbal/written advice to give. They were informed of the ENT departmental epistaxis advice sheets that were made available in the A\&E department. Copies of the epistaxis advice sheet, which includes a comprehensive list of behaviour to avoid after a bleed, were distributed to all doctors present. The practice regarding advice and the re-attendance rates were re-audited in May/ June 2003.

\section{RESULTS}

During the first phase of the audit, 99 patients presented to the A\&E department with epistaxis. The male:female ratio was 56:43 and the age range was 3-84 years. The causes of epistaxis were varied and were most commonly anticoagulant related. Other causes were hypertension, trauma, and allergic and infective rhinitis, and some cases were idiopathic.

In March and April 2003, 63 of the 99 patients presenting with epistaxis were seen and discharged by A\&E doctors. The remaining 36 were also seen by ENT doctors. Of those seen only by A\&E doctors, $19 \%$ received verbal advice and $2 \%$ received written advice. These results were complemented by the A\&E senior house officer questionnaire.

There were 12 re-attenders during the first cycle; 11 had previously been seen only by an A\&E doctor, and none had been given verbal or written advice. The remaining patient had seen an ENT doctor during the previous attendance. In total, twp patients seen by ENT doctors re-attended with recurrent bleeds within the whole duration of the audit cycle, and both had received advice sheets.

The number of patients presenting with epistaxis during the re-audit period who had been seen only by A\&E doctors was similar to the number presenting in the first half of the audit (63 and 60). The results after the re-audit showed a significant increase in the proportion of patients receiving advice by A\&E doctors. Verbal and written advice increased from $19 \%$ to $61 \%$ and $2 \%$ to $54 \%$ respectively.

The number of re-attenders who had previously only seen A\&E doctors was reduced from $11(17 \%)$ in the first half to 5 $(8 \%)$ in the second half of the cycle, a 9\% reduction. All patients re-attending in the second half were given advice sheets.

\section{CONCLUSION}

Data from the retrospective audit by $\mathrm{O}^{\prime}$ Donnell ${ }^{3}$ suggest that the incidence of patients with a primary diagnosis of epistaxis presenting to A\&E is 108 per 100000 population annually. The incidence of those requiring admission or specialist treatment for epistaxis is reported as between 26.4 and 28.8

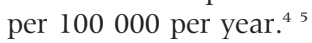

Most epistaxis patients (63\%) presenting to the A\&E department at St George's Hospital are seen only by a casualty doctor. It is therefore essential that these doctors are given adequate training in the management and prevention of epistaxis, as this may reduce the number of re-attenders.

Abbreviations: A\&E, accident and emergency; ENT, ear, nose, and throat 
These results indicate that at least $80 \%$ of epistaxis patients seen and discharged by casualty doctors in A\& E receive little or no information regarding the various activities that may trigger a further bleed. Consequently, these patients are unaware that these activities must be avoided and may be at greater risk of subsequent bleeds.

We feel that the provision of information leaflets and verbal advice to patients with epistaxis may be important in the prevention of further bleeds and subsequent hospital attendances. This is the first published study addressing this topic. Clear advice in this group of patients can easily be given and may aid in reduction in $\mathrm{A} \& \mathrm{E}$ attendance.

\section{Authors' affiliations}

N Eze, S Lo, A Toma, Department of Otolaryngology-Head and Neck

Surgery, St George's Hospital Medical School, University of London, UK

Competing interests: none declared
Correspondence to: Miss N Eze, Department of Otolaryngology, Great Ormond Street Hospital, London WCIN 3JH, UK; nnekaeze@yahoo. co.uk

Accepted for publication 15 July 2004

\section{REFERENCES}

1 Lavy JA, Koay CB. First aid treatment of epistaxis - are the patients well informed? J Acc Emerg Med 1996;13:193-5.

2 Murthy P, Christodoulou C, Yatigammana N, et al. The influence of medical audit on the management of epistaxis in three district general hospitals. $J$ Laryngol Otol 1994;108:38-41.

3 O'Donnell M, Robertson G, McGary GW. A new bipolar diathermy probe for the outpatient management of adult acute epistaxis. Clin Otol 1999:24:537-41.

4 Wild DC, Spraggs PD. Treatment of epistaxis in accident and emergency departments in the UK. J Laryngol Otol 2002;116:597-601.

5 O'Driscoll K, Donnelly MJ, McShane DP, et al. An audit of the ENT casualty service at the Royal Victoria Eye and Ear Hospital. Ir J Med Sci $1993 ; 162: 462-5$ 